\title{
Co-occurrence of hearing loss and posttraumatic stress disorder among injured military personnel: a retrospective study
}

Andrew J. MacGregor ${ }^{1 *}$, Antony R. Joseph ${ }^{2,3}$, G. Jay Walker ${ }^{1,3}$ and Amber L. Dougherty ${ }^{1,3}$

\begin{abstract}
Background: Posttraumatic stress disorder (PTSD) and hearing loss are hallmark public health issues related to military service in Iraq and Afghanistan. Although both are significant individual contributors to disability among veterans, their co-occurrence has not been specifically explored.

Methods: A total of 1179 male U.S. military personnel who sustained an injury between 2004 and 2012 during operations in Iraq or Afghanistan were identified from clinical records. Pre- and postinjury audiometric data were used to define new-onset hearing loss, which was categorized as unilateral or bilateral. Diagnosed PTSD was abstracted from electronic medical records. Logistic regression analysis examined the relationship between hearing loss and PTSD, while adjusting for age, year of injury, occupation, injury severity, injury mechanism, and presence of concussion.

Results: The majority of the study sample were aged 18-25 years (79.9\%) and sustained mild-moderate injuries (94.6\%). New-onset hearing loss was present in 14.4\% of casualties (10.3\% unilateral, 4.1\% bilateral). Rates of diagnosed PTSD were 9.1, 13.9, and 29.2\% for those with no hearing loss, unilateral hearing loss, and bilateral hearing loss, respectively. After adjusting for covariates, those with bilateral hearing loss had nearly three-times higher odds of PTSD (odds ratio $=2.92 ; 95 \% \mathrm{Cl}, 1.47-5.81$ ) compared to those with no hearing loss. Unilateral hearing loss was not associated with PTSD.

Conclusions: Both PTSD and hearing loss are frequent consequences of modern warfare that adversely affect the overall health of the military. Bilateral, but not unilateral, hearing loss was associated with a greater burden of PTSD. This has implications for warfighter rehabilitation and should encourage collaboration between audiology and mental health professionals.
\end{abstract}

Keywords: Hearing loss, PTSD, Military, Veteran

\section{Background}

Posttraumatic stress disorder (PTSD) is a frequent wartime problem [1-3]. The disorder is characterized by

\footnotetext{
* Correspondence: andrewmacgregor@protonmail.com

${ }^{1}$ Medical Modeling, Simulation, and Mission Support Department, Naval Health Research Center, 140 Sylvester Road, San Diego, CA, USA

Full list of author information is available at the end of the article
}

anxiety, detachment, avoidance behaviors, and intrusive thoughts in response to a traumatic event [4]. The post9/11 military conflicts in Iraq and Afghanistan produced a significant PTSD health burden, resulting in increased rates of hospitalization, a surge in disability claims, and an array of comorbid physical and mental health conditions [5-9]. With military members eventually

(C) The Author(s). 2020 Open Access This article is licensed under a Creative Commons Attribution 4.0 International License, which permits use, sharing, adaptation, distribution and reproduction in any medium or format, as long as you give appropriate credit to the original author(s) and the source, provide a link to the Creative Commons licence, and indicate if changes were made. The images or other third party material in this article are included in the article's Creative Commons licence, unless indicated otherwise in a credit line to the material. If material is not included in the article's Creative Commons licence and your intended use is not permitted by statutory regulation or exceeds the permitted use, you will need to obtain permission directly from the copyright holder. To view a copy of this licence, visit http://creativecommons.org/licenses/by/4.0/ The Creative Commons Public Domain Dedication waiver (http://creativecommons.org/publicdomain/zero/1.0/) applies to the data made available in this article, unless otherwise stated in a credit line to the data. 
transitioning to civilian life, the effects of PTSD become a wider public health issue [10].

Similar to PTSD, hearing loss is a significant public health problem in the military, as the Department of Veterans Affairs (VA) reported that the cost of hearing loss exceeds one billion dollars annually [11]. In general, military personnel have a higher risk of hearing loss than their civilian counterparts [12]. During a military wartime deployment, there are multiple risk factors for hearing loss, including repeated occupational noise exposures as well as blast exposure [13]. Asymmetrical warfare employed by the enemy was prevalent in Iraq and Afghanistan (e.g., improvised explosive devices), which resulted in blasts causing the majority of all injuries $[14,15]$. It is well established that exposure to blasts leads to adverse effects on the auditory system [16-19]. A recent retrospective cohort study found a more than two-fold increase in risk of hearing loss among military personnel with blast relative to nonblast injuries [19].

Although PTSD and hearing loss both contribute significantly to the overall health burden in the military, their co-occurrence has not been widely described in the literature [20]. A VA study by Swan and colleagues was the first to suggest the association, finding that those with a diagnosis of hearing loss were more likely to have a diagnosis of PTSD [21]. This study used diagnostic codes to identify hearing loss and was unable to achieve the level of granularity found with audiometric data. One civilian study found a higher rate of anxiety disorder in a community sample with sensorineural hearing loss compared with controls, but PTSD was not specifically explored [22]. Further, hearing loss and PTSD have been individually linked to physical injury [19, 23], most notably blast-related concussion, a novel injury type of increased military importance over the course of the Iraq and Afghanistan conflicts [24, 25].

The paucity of literature on hearing loss and PTSD, in addition to the combined public health impacts, warrants an investigation of their co-occurrence. The present study aimed to examine the relationship between new-onset hearing loss and PTSD, as measured using audiometric data, among a sample of U.S. military personnel with deployment-related injury. It was hypothesized that hearing loss would be positively associated with PTSD diagnosis.

\section{Methods}

\section{Study sample}

Military personnel injured during combat deployment in Iraq and Afghanistan between 2004 and 2012 were identified from the Expeditionary Medical Encounter Database (EMED), which contains clinical encounters across all levels of medical care [26]. The EMED clinical record has provider notes and information that describe the injury event, including mechanism and severity, which is then verified by certified nurse coders. Data extracted from the EMED can be connected with other Department of Defense (DoD) medical databases (e.g., Military Health System Data Repository [MDR]), as well as tactical, personnel, operational, and deployment-related data.

A subset of the EMED was used to create the BlastRelated Auditory Injury Database (BRAID), which includes injured military personnel, specifically Navy and Marine Corps, with valid audiometric information from the DoD Hearing Conservation Program [27]. Details regarding the creation of the BRAID from the EMED have been published elsewhere [18]. The BRAID includes service members with blast- and nonblast-related injuries sustained during deployment. Due to small numbers of women in the BRAID, only men were included in this analysis. For inclusion, per previous research on the BRAID, individuals in the present study had audiometric data within 1 year preinjury and 1 year postinjury $(n=$ 1573). After excluding those with a previous PTSD diagnosis $(n=6)$ and those with preinjury hearing loss $(n=$ 388), the final study sample consisted of 1179 individuals. This study received local Institutional Review Board approval.

\section{Variables and measures \\ Demographic characteristics}

Included age at time of injury, which was calculated as the difference between date of injury and birth and categorized as $18-25$ or 26 and older. Occupation was dichotomized per DoD Military Occupational Specialty code into infantry or non-infantry [28].

\section{Injury-related characteristics}

Were abstracted from the EMED clinical record. Year of injury was categorized as 2004-2006 or 2007-2012. Battle injury was categorized as battle or nonbattle, and blast injury as blast or nonblast. Injuries were distinguished by battle and blast injury in order to account for combat noise exposure. Injury severity was measured using the Injury Severity Score (ISS), which provides an overall measure of injury severity for individuals with multiple injuries $[29,30]$. The ISS is calculated using Abbreviated Injury Scale scores [31] ranging from 0 (no injury) to 75 (unsurvivable injury). In the present study, ISS was categorized as 1-8 (i.e., mild-moderate injury severity) or 9 and greater (i.e., serious injury and worse) [29-31]. Concussion was indicated if documented in the EMED clinical record at the time of injury.

\section{Hearing loss}

Was measured using audiometric pure-tone hearing threshold levels for left and right ears at six test 
frequencies $(500 \mathrm{~Hz}, 1000 \mathrm{~Hz}, 2000 \mathrm{~Hz}, 3000 \mathrm{~Hz}, 4000$ $\mathrm{Hz}$, and $6000 \mathrm{~Hz}$ ) that were abstracted from the BRAID. Postinjury hearing loss was defined as a response exceeding $25 \mathrm{~dB}$ at any test frequency [19]. Postinjury hearing loss was further categorized into unilateral if present in only one ear or bilateral if present in both ears.

\section{PTSD}

Diagnosis was obtained from outpatient medical information in the MDR. PTSD was defined as presence of an International Classification of Diseases, 9th Revision, Clinical Modification code of 309.81 within 2 years postinjury [32].

\section{Statistical analyses}

Data management and statistical analyses were performed using SAS software, version 9.4 (SAS Institute, Inc., Cary, North Carolina). Characteristics of the study sample were stratified by hearing loss status and chi- square statistics were used to test for differences. Tetrachoric correlation coefficients were calculated to highlight relationships between covariates. Univariate and multivariate logistic regression with backward selection was used to model the relationship between hearing loss and PTSD. The Hosmer-Lemeshow test was used to assess the model for goodness of fit with an alpha level of 0.10 .

\section{Results}

Overall, unilateral and bilateral hearing loss was present in $10.3 \%(n=122)$ and $4.1 \%(n=48)$ of study participants, respectively. Sample characteristics are shown in Table 1 . The majority were aged $18-25$ years at time of injury, in non-infantry positions, and injured between the years 2004 and 2006. Nonblast and nonbattle injuries predominated, and concussion was present in $26.2 \%$ of personnel. Among those with bilateral hearing loss, $72.9 \%$ were injured by a blast compared with only $37.5 \%$ for those with no hearing loss. Rates of hearing loss were

Table 1 Descriptive characteristics of the study sample

\begin{tabular}{|c|c|c|c|c|c|}
\hline Variable & $\begin{array}{l}\text { Total } \\
\text { No. (\%) } \\
(n=1179)\end{array}$ & $\begin{array}{l}\text { No hearing loss } \\
\text { No. }(\%) \\
(n=1009)\end{array}$ & $\begin{array}{l}\text { Unilateral hearing loss } \\
\text { No. }(\%) \\
(n=122)\end{array}$ & $\begin{array}{l}\text { Bilateral hearing loss } \\
\text { No. }(\%) \\
(n=48)\end{array}$ & $p$-value \\
\hline Age, years & & & & & 0.793 \\
\hline $18-25$ & $942(79.9)$ & 806 (79.9) & $96(78.7)$ & $40(83.3)$ & \\
\hline 26 and older & $237(20.1)$ & $203(20.1)$ & $26(21.3)$ & $8(16.7)$ & \\
\hline Year of injury & & & & & $<0.001$ \\
\hline 2004-2006 & 765 (64.9) & $677(67.1)$ & $67(54.9)$ & $21(43.8)$ & \\
\hline $2007-2012$ & $414(35.1)$ & 332 (32.9) & $55(45.1)$ & $27(56.3)$ & \\
\hline Occupation & & & & & 0.003 \\
\hline Non-infantry & $603(51.2)$ & $536(53.1)$ & $46(37.7)$ & $21(43.8)$ & \\
\hline Infantry & $576(48.9)$ & $473(46.9)$ & $76(62.3)$ & $27(56.3)$ & \\
\hline Battle injury & & & & & $<0.001$ \\
\hline No & $614(52.1)$ & $557(55.2)$ & $44(36.1)$ & $13(27.1)$ & \\
\hline Yes & $565(47.9)$ & $452(44.8)$ & 78 (63.9) & $35(72.9)$ & \\
\hline Blast injury & & & & & $<0.001$ \\
\hline No & 699 (59.3) & $631(62.5)$ & $55(45.1)$ & $13(27.1)$ & \\
\hline Yes & $480(40.7)$ & $378(37.5)$ & $67(54.9)$ & $35(72.9)$ & \\
\hline ISS & & & & & 0.647 \\
\hline $1-9$ & 1115 (94.6) & 956 (94.8) & 115 (94.3) & $44(91.7)$ & \\
\hline 9 and greater & $64(5.4)$ & $53(5.3)$ & $7(5.7)$ & $4(8.3)$ & \\
\hline Concussion & & & & & 0.002 \\
\hline No & $870(73.8)$ & $760(75.3)$ & $84(68.9)$ & $26(54.2)$ & \\
\hline Yes & $309(26.2)$ & $249(24.7)$ & $38(31.2)$ & $22(45.8)$ & \\
\hline PTSD & & & & & $<0.001$ \\
\hline No & 1056 (89.6) & 917 (90.9) & $105(86.1)$ & $34(70.8)$ & \\
\hline Yes & $123(10.4)$ & $92(9.1)$ & $17(13.9)$ & 14 (29.2) & \\
\hline
\end{tabular}


significantly higher in infantry personnel $(p=0.003)$, and those with battle injury $(p<0.001)$, blast injury $(p<$ $0.001)$, and concussion $(p=0.002)$. Hearing loss groups did not statistically differ by age or ISS ( $p s>0.05)$. The overall rate of PTSD was $10.4 \%$, and rates differed significantly across hearing loss groups $(p<0.001)$. Those with bilateral hearing loss had the highest PTSD rate at $29.2 \%$, followed by unilateral hearing loss at $13.9 \%$, and no hearing loss at $9.1 \%$.

The tetrachoric correlation matrix for covariates is shown in Table 2. The highest correlations were between battle injury and blast injury (0.994), blast injury and concussion (0.851), and battle injury and concussion (0.798). Notably, only one nonbattle injury was the result of a blast mechanism, and $57.7 \%$ of blast injuries resulted in concussion compared with only $4.6 \%$ of nonblast injuries. The lowest correlations were between ISS and year of injury (0.011), and ISS and age (0.031).

Table 3 details the results of univariate and multivariate logistic regression modeling. In univariate regression, all variables except for age were significantly associated with PTSD diagnosis $(p s<0.01)$. In multivariate modeling with backward selection, however, only battle injury (odds ratio $[\mathrm{OR}]=6.64 ; 95 \%$ confidence interval $[\mathrm{CI}]$, $4.00-11.03)$ and bilateral hearing loss $(\mathrm{OR}=2.92$; $95 \%$ CI, 1.47-5.81) were significantly associated with PTSD. Unilateral hearing loss was not significantly associated with PTSD (OR $=1.23$; 95\% CI, 0.69-2.17). The Hosmer Lemeshow test had a $p$-value greater than 0.10 , indicating a good model fit.

\section{Discussion}

Hearing loss and PTSD are significant public health problems in the military. The present study suggests the two may be linked. In a large group of injured male military personnel, we found that the odds of PTSD are approximately three-times higher in individuals with postinjury bilateral hearing loss when compared to personnel without hearing loss. Hearing loss can affect listening performance and decrease quality of life, which may serve to exacerbate mental health problems such as PTSD [33].
Table 3 Univariate and multivariate ${ }^{a}$ logistic regression analysis of hearing loss and PTSD $(n=1179)$

\begin{tabular}{|c|c|c|}
\hline \multirow[t]{3}{*}{ Variable } & \multicolumn{2}{|l|}{ PTSD } \\
\hline & Univariate & Multivariate \\
\hline & OR $(95 \% \mathrm{Cl})$ & OR $(95 \% \mathrm{Cl})$ \\
\hline \multicolumn{3}{|l|}{ Hearing loss } \\
\hline None & Ref & Ref \\
\hline Unilateral & $1.61(0.93-2.81)$ & $1.23(0.69-2.17)$ \\
\hline Bilateral & $4.11(2.13-7.93)^{b}$ & $2.92(1.47-5.81)^{b}$ \\
\hline Age, years & & NS \\
\hline $18-25$ & Ref & \\
\hline 26 and older & $0.70(0.42-1.17)$ & \\
\hline Year of injury & & NS \\
\hline 2004-2006 & Ref & \\
\hline 2007-2012 & $1.57(1.08-2.29)^{b}$ & \\
\hline Occupation & & NS \\
\hline Non-infantry & Ref & \\
\hline Infantry & $2.47(1.66-3.67)^{\mathrm{b}}$ & \\
\hline \multicolumn{3}{|l|}{ Battle injury } \\
\hline No & Ref & Ref \\
\hline Yes & $7.07(4.27-11.69)^{b}$ & $6.64(4.00-11.03)^{b}$ \\
\hline Blast injury & & NS \\
\hline No & Ref & \\
\hline Yes & $4.66(3.07-7.07)^{b}$ & \\
\hline ISS & & NS \\
\hline $1-8$ & Ref & \\
\hline 9 and greater & $2.86(1.55-5.26)^{b}$ & \\
\hline Concussion & & NS \\
\hline No & Ref & \\
\hline Yes & $2.86(1.96-4.19)^{\mathrm{b}}$ & \\
\hline
\end{tabular}

Cl confidence interval, ISS Injury Severity Score, NS not selected, OR odds ratio, PTSD posttraumatic stress disorder, Ref reference level

aultivariate model employed backward selection and was indicated as a good fit by the Hosmer-Lemeshow test $(p>0.10)$ ${ }^{\mathrm{b}_{p}}<0.01$

Physical injury during combat is an established predictor of PTSD [23]. Koren et al. found that injured soldiers who experienced combat events similar to those of

Table 2 Tetrachoric correlation matrix for covariates $(n=1179)$

\begin{tabular}{|c|c|c|c|c|c|c|c|}
\hline Variable & Age & Year of injury & Infantry & Battle injury & Blast injury & ISS & Concussion \\
\hline Age & 1.000 & - & - & - & - & - & - \\
\hline Year of injury & 0.039 & 1.000 & - & - & - & - & - \\
\hline Infantry & -0.226 & 0.276 & 1.000 & - & - & - & - \\
\hline Battle injury & -0.141 & 0.357 & 0.683 & 1.000 & - & - & - \\
\hline Blast injury & -0.146 & 0.334 & 0.584 & 0.994 & 1.000 & - & - \\
\hline ISS & 0.031 & 0.011 & 0.201 & 0.473 & 0.249 & 1.000 & - \\
\hline Concussion & -0.176 & 0.486 & 0.464 & 0.798 & 0.851 & 0.095 & 1.000 \\
\hline
\end{tabular}


a noninjured control group were nearly nine-times more likely to develop PTSD [34]. Several studies have demonstrated that certain types of injuries may cause individuals to recall a traumatic event [35-37], including those with injuries that cause more severe physical problems, or impact appearance in daily life such as burns and facial trauma. It is possible that these injuries might exacerbate psychological effects of the traumatic event, thus increasing the chance and/or frequency of recollection, which in turn elevates the risk of PTSD. Extending this hypothesis, sensory injuries such as hearing loss can also adversely affect the life of working-aged adults; hearing loss acquired in adulthood has been found to lead to adjustment issues both personally and professionally [38]. It can result in mental distress due to social isolation, which can have a significant impact on personal relationships [39, 40]. In an occupational setting, workers with hearing loss report a greater sensitivity to background noise, communication issues that disrupt their work, and unsupportive supervisors [41, 42]. Injury-related hearing loss presents as an abrupt loss, which can leave the patient ill-prepared to deal with adjustment issues [43]. In summary, if an injury results in impairment that impacts daily life to a noticeable degree, whether that impairment is physical or sensory, the injury incident may be recalled more regularly, and thus resolution of the trauma might be more intractable. Studies using survey designs in clinical samples are needed to further explore this pathway.

Comorbidities of both PTSD and hearing loss may have played a role in the observed association. Depression has been frequently linked to PTSD and multiple studies have found increased rates of depression among those with hearing loss $[33,43,44]$. Abrams et al. found a strong correlation between hearing loss and depression in older veterans and concluded that individuals with both conditions had lowered measures of health-related quality of life [33]. Similarly, another study found that patients with abrupt or sudden hearing loss had a more than two times higher risk for developing depressive disorders than those without hearing loss [43]. Further, tinnitus commonly co-occurs with noise-induced hearing loss and has been associated with PTSD [45-47]. One study among a refugee population identified a significantly higher rate of PTSD among those with tinnitus and the study authors posited that tinnitus impacted trauma recall and flashbacks [46]. In addition, a study among military veterans found a correlation between PTSD and tinnitus, and reported that sounds triggering exacerbation of tinnitus similarly affected PTSD symptom severity [47]. Altogether, these findings indicate that longitudinal studies with multiple data ascertainment points are needed to investigate the effect of tinnitus and depression on the relationship between PTSD and hearing loss.
The present study has several strengths. In comparison with the recent study by Swan et al. that identified a relationship between PTSD and hearing loss using strictly diagnosis codes, the present study used audiometric data collected under standardized medical surveillance protocols [21]. The use of audiometric data to identify at-risk groups for PTSD and other mental health disorders has been investigated previously with mixed findings [4850]. The results of this study may further encourage collaboration between audiologists and mental health professionals, particularly at military and VA hospitals where combat veterans are likely to be seen. The present study was also strengthened by the stratification of hearing loss into unilateral and bilateral, which added granularity to the results. In contrast to a previous study by Mahapatra that found no difference between bilateral and unilateral hearing loss with relation to depression, we found that those with bilateral hearing loss, but not unilateral, had a higher odds of PTSD [51]. Bilateral and unilateral hearing loss have different clinical profiles and further investigation is needed to determine what specific hearing difficulties (e.g., communication deficiencies, sound localization problems) have the greatest psychological impact [52]. Using the BRAID offers a unique examination of hearing loss in a population of mostly young adults. By using validated pre- and postinjury audiograms, we were able to capture hearing loss that occurred in proximity to the injury event. Last, the ability to link the BRAID to electronic medical records allowed for the abstraction of provider-diagnosed PTSD, thus avoiding the information bias issues found with self-reported data.

There are limitations that warrant mention. First, regarding the temporal relationship between hearing loss and PTSD, both were ascertained at different times postinjury. Using this methodology, it is possible that a diagnosis of PTSD might be recorded before or after the date of the audiogram and, as such, temporality could not be established. Using diagnosis codes to ascertain PTSD also excludes those individuals who may meet criteria for the disorder, but do not present for care. Moreover, a diagnosis of PTSD could be delayed by years, thus a diagnostic bias may occur with follow-up time restricted to two years. It is also possible that hearing loss could worsen between the blast injury and audiogram date, sometimes unrelated to the injury. A full assessment of combat experiences for each individual was not available for the present study. While it is arguable that a blast produced the hearing loss, it is likely that a collection of combat exposures caused the PTSD, the blast event being one. Though we accounted for injuryspecific variables in our analysis, it may be difficult to fully adjust for the possible confounding effects of trauma severity on hearing loss. Additional controlled 
studies are needed to clarify these effects. Restricting the study sample to men precluded any examination of possible sex differences, and the findings may not generalize to military women. Lastly, potential comorbidities, such as tinnitus and depression, were not measured in the present study.

\section{Conclusions}

To our knowledge, this is the first study to examine the association between PTSD and hearing loss in military personnel that have been injured during combat deployment. Taken together, both conditions are responsible for billions in health care costs for veterans. Delays in identification of these conditions may result in reduced quality of life for affected individuals, thus early provider diagnosis and intervention may be highly beneficial. Approximately $80 \%$ of primary care providers do not screen for hearing loss, and individuals with hearing loss often do not recognize a problem until they are examined and counseled [41]. This further encourages a strong working relationship between audiology and mental health professionals because military personnel with bilateral hearing loss should be monitored for possible development of PTSD. Future research should focus on determining etiological pathways and mediating factors, such as tinnitus and depression, and whether successful treatment of one condition has an impact on the other. Injuries of modern warfare can have serious audiological and psychological consequences, and health intervention strategies are paramount to maximize individual quality of life and overall military readiness.

\section{Abbreviations \\ BRAID: Blast-Related Auditory Injury Database; Cl: confidence interval; DoD: Department of Defense; EMED: Expeditionary Medical Encounter Database; ISS: Injury Severity Score; MDR: Military Health System Data Repository; OR: odds ratio; PTSD: posttraumatic stress disorder; \\ VA: Department of Veterans Affairs}

\section{Acknowledgements}

The authors thank the epidemiologists and data analysts in the Analytics and Epidemiology Support for Operational Populations Division at Naval Health Research Center for their assistance with this project.

\section{Disclaimer}

I am a military service member or employee of the U.S. Government. This work was prepared as part of my official duties. Title 17, U.S.C. \$105 provides that copyright protection under this title is not available for any work of the U.S. Government. Title 17, U.S.C. \$101 defines a U.S. Government work as work prepared by a military service member or employee of the U.S. Government as part of that person's official duties. Report \#20-17 was supported by the U.S. Navy Bureau of Medicine and Surgery under work unit no. 60808. The views expressed in this article are those of the authors and do not necessarily reflect the official policy or position of the Department of the Navy, Department of Defense, nor the U.S. Government. The study protocol was approved by the Naval Health Research Center Institutional Review Board in compliance with all applicable Federal regulations governing the protection of human subjects. Research data were derived from an approved Naval Health Research Center Institutional Review Board protocol, number NHRC.2003.0025.

\section{Authors' contributions}

AJM conceptualized the study idea and was involved in all phases of analysis, interpretation, and writing; ARJ provided subject matter expertise on audiology and assisted with analysis, interpretation, and writing; GJW was the primary data analyst on the study; ALD provided subject matter expertise and assisted with data interpretation and scientific writing. All authors critically reviewed the manuscript for important intellectual content and approved the final version.

\section{Funding}

This work was supported by the U.S. Navy Bureau of Medicine and Surgery under work unit no. 60808.

\section{Availability of data and materials}

The data sets generated and/or analyzed during the current study are not publicly available due to personally identifiable information regulations, but may be made available by the corresponding author upon reasonable request and approval by the Naval Health Research Center Institutional Review Board.

\section{Ethics approval and consent to participate}

The study protocol was approved by the Naval Health Research Center Institutional Review Board in compliance with all applicable Federal regulations governing the protection of human subjects. Research data were derived from an approved Naval Health Research Center Institutional Review Board protocol, number NHRC.2003.0025.

\section{Consent for publication}

Not applicable.

\section{Competing interests}

The authors declare that they have no competing interests.

\section{Author details}

${ }^{1}$ Medical Modeling, Simulation, and Mission Support Department, Naval Health Research Center, 140 Sylvester Road, San Diego, CA, USA. ${ }^{2}$ Hearing Loss Prevention Laboratory, Communication Sciences and Disorders Department, Illinois State University, Normal, IL, USA. 'Leidos, Inc., San Diego, CA, USA.

Received: 26 March 2020 Accepted: 27 May 2020

Published online: 08 July 2020

\section{References}

1. Hyams KC, Wignall FS, Roswell R. War syndromes and their evaluation: from the U.S. civil war to the Persian Gulf war. Ann Intern Med. 1996;125(5):398405.

2. Boyle CA, Decouflé $P, O^{\prime}$ Brien TR. Long-term health consequences of military service in Vietnam. Epidemiol Rev. 1989;11:1-27.

3. Stimpson NJ, Thomas HV, Weightman AL, Dunstan F, Lewis G. Psychiatric disorder in veterans of the Persian Gulf war of 1991. Br J Psychiatry. 2003; 182:391-403.

4. Friedman MJ. Post-traumatic and acute stress disorders: the latest assessment and treatment strategies. 4th ed. Kansas City: Compact Clinicals; 2006.

5. Armed Forces Health Surveillance Center. Summary of mental disorder hospitalizations, active and reserve components, U.S. Armed forces, 20002012. MSMR. 2013;20(7):4-11.

6. McNally RJ, Frueh BC. Why are Iraq and Afghanistan war veterans seeking PTSD disability compensation at unprecedented rates? J Anxiety Disord. 2013;27(5):520-6.

7. Seal $\mathrm{KH}$, Bertenthal $\mathrm{D}$, Miner CR, Sen S, Marmar C. Bringing the war back home: mental health disorders among 103,788 US veterans returning from Iraq and Afghanistan seen at Department of Veterans Affairs facilities. Arch Intern Med. 2007;167(5):476-82.

8. Ramchand R, Rudavsky R, Grant S, Tanielian T, Jaycox L. Prevalence of, risk factors for, and consequences of posttraumatic stress disorder and other mental health problems in military populations deployed to Iraq and Afghanistan. Curr Psychiatry Rep. 2015;17:37.

9. El-Gabalawy R, Blaney C, Tsai J, Sumner JA, Pietrzak RH. Physical health conditions associated with full and subthreshold PTSD in U.S. military 
veterans: results from the national health and resilience in veterans study. J Affect Disord. 2018;227:849-53.

10. Hoge CW, Auchterlonie JL, Milliken CS. Mental health problems, use of mental health services, and attrition from military service after returning from deployment to Iraq or Afghanistan. JAMA. 2006;295(9):1023-32.

11. U.S. Department of Veterans Affairs. Veterans Benefits Administration. Annual Benefits Report Fiscal Year 2012. Washington, DC: Department of Veterans Affairs; 2013. https://www.benefits.va.gov/REPORTS/abr/archive.asp. Accessed 7 Oct 2019.

12. Centers for Disease Control and Prevention. Severe hearing impairment among military veterans--United States, 2010. MMWR Morb Mortal Wkly Rep. 2011;60(28):955-8.

13. Joseph AR, Horton JL, Clouser MC, MacGregor AJ, Louie M, Galarneau MR. Development of a comprehensive blast-related auditory injury database (BRAID). J Rehabil Res Dev. 2016:53(3):295-306.

14. Wallace D. Improvised explosive devices and traumatic brain injury: the military experience in Iraq and Afghanistan. Australas Psychiatry. 2009;17(3): 218-24

15. Greer N, Sayer N, Kramer M, Koeller E, Velasquez T. Prevalence and epidemiology of combat blast injuries from the military cohort 2001-2014. Washington, DC: Department of Veterans Affairs; 2016.

16. Van Campen LE, Dennis JM, Hanlin RC, King SB, Velderman AM. One-year audiologic monitoring of individuals exposed to the 1995 Oklahoma City bombing. J Am Acad Audiol. 1999;10(5):231-47.

17. Nageris Bl, Attias J, Shemesh R. Otologic and audiologic lesions due to blast injury. J Basic Clin Physiol Pharmacol. 2008;19(3-4):185-91.

18. Dougherty AL, MacGregor AJ, Han PP, Viirre E, Heltemes KJ, Galarneau MR. Blast-related ear injuries among U.S. military personnel. J Rehabil Res Dev. 2013;50(6):893-904.

19. Joseph AR, Shaw JL, Clouser MC, MacGregor AJ, Galarneau MR. Impact of blast injury on hearing in a screened male military population. Am J Epidemiol. 2018;187(1):7-15.

20. Shoham N, Lewis G, Favarato G, Cooper C. Prevalence of anxiety disorders and symptoms in people with hearing impairment: a systematic review. Soc Psychiatry Psychiatr Epidemiol. 2019;54(6):649-60.

21. Swan AA, Nelson JT, Swiger B, Jaramillo CA, Eapen BC, Packer M, et al. Prevalence of hearing loss and tinnitus in Iraq and Afghanistan veterans: a chronic effects of Neurotrauma consortium study. Hear Res. 2017;349:4-12.

22. Hsu WT, Hsu CC, Wen MH, Lin HC, Tsai HT, Su P, et al. Increased risk of depression in patients with acquired sensory hearing loss: a 12-year followup study. Medicine. 2016;95(44):e5312.

23. MacGregor AJ, Tang JJ, Dougherty AL, Galarneau MR. Deployment-related injury and posttraumatic stress disorder in US military personnel. Injury. 2013:44(11):1458-64.

24. Halbauer JD, Ashford JW, Zeitzer JM, Adamson MM, Lew HL, Yesavage JA. Neuropsychiatric diagnosis and management of chronic sequelae of warrelated mild to moderate traumatic brain injury. J Rehabil Res Dev. 2009; 46(6):757-96.

25. Scofield DE, Proctor SP, Kardouni JR, Hill OT, McKinnon CJ. Risk factors for mild traumatic brain injury and subsequent post-traumatic stress disorder and mental health disorders among United States Army soldiers. J Neurotrauma. 2017;34:3249-55.

26. Galarneau MR, Hancock WC, Konoske P, Melcer T, Vickers RR, Walker GJ, et al. The navy-marine corps combat trauma registry. Mil Med. 2006;171: 691-7.

27. U.S. Department of Defense, Office of the Under Secretary of Defense for Personnel and Readiness. DoD Instruction 6055.12. Hearing Conservation Program (HCP). 2019. https://www.esd.whs.mil/Portals/54/Documents/DD/ issuances/dodi/605512p.pdf?ver=2017-10-25-110159-777. Accessed 7 Oct 2019.

28. U.S. Department of Defense, Office of the Under Secretary of Defense, Personnel and Readiness. DoD Instruction 1312.1-1. Occupational Conversion Index. 1997. https://apps.dtic.mil/dtic/tr/fulltext/u2/a327949.pdf. Accessed 26 Sept 2019.

29. Baker SP, O'Neill B, Haddon W Jr, Long WB. The injury severity score: a method for describing patients with multiple injuries and evaluating emergency care. J Trauma. 1974;14:187-96.

30. Copes WS, Champion HR, Sacco WJ, Lawnick MM, Keast SL, Bain LW. The injury severity score revisited. J Trauma. 1988;28:69-77.

31. Gennarelli TA, Wodzin E. AIS 2005: a contemporary injury scale. Injury. 2006; 37:1083-91.
32. Commission on Professional Hospital Activities. International classification of diseases, 9th revision, clinical modification. Ann Arbor: Edwards Brothers; 1977.

33. Abrams TE, Barnett MJ, Hoth A, Schultz S, Kaboli PJ. The relationship between hearing impairment and depression in older veterans. J Am Geriatr Soc. 2006;54(9):1475-7.

34. Koren D, Norman D, Cohen A, Berman J, Klein EM. Increased PTSD risk with combat-related injury: a matched comparison study of injured and uninjured soldiers experiencing the same combat events. Am J Psychiatry. 2005;162(2):276-82.

35. Grieger TA, Cozza SJ, Ursano RJ, Hoge C, Martinez PE, Engel CC, Wain HJ. Posttraumatic stress disorder and depression in battle-injured soldiers. Am J Psychiatry. 2006;163(10):1777-83.

36. Madianos MG, Papaghelis M, loannovich J, Dafni R. Psychiatric disorders in burn patients: a follow-up study. Psychother Psychosom. 2001;70(1):30-7.

37. Fukunishi I. Relationship of cosmetic disfigurement to the severity of posttraumatic stress disorder in burn injury or digital amputation. Psychother Psychosom. 1999;68(2):82-6.

38. National Research Council Committee on Disability Determination for Individuals with Hearing Impairments. In: Dobie RA, Van Hemel S, editors. Hearing loss: determining eligibility for Social Security benefits. Washington, DC: National Academies Press; 2005.

39. Meadow-Orlans KP. Social and psychological effects of hearing loss in adulthood: a literature review. In: Orlans H, editor. Adjustment to adult hearing loss. San Diego: College-Hill Press; 1985. p. 35-57.

40. Schein S, Bottum EB, Lawler JT, Madory R, Wantuch E. Psychological challenges encountered by hearing impaired adults and their families. Rehabil Psychol. 2001;46:322-3.

41. Laroche C, Garcia LJ, Barrette J. Perceptions by persons with hearing impairment, audiologists, and employers of the obstacles to work integration. J Acad Rehabil Audiol. 2000;23:63-90.

42. Schroedel JG, Geyer PD. Long-term career attainments of deaf and hard of hearing college graduates: results from a 15-year follow-up survey. Am Ann Deaf. 2000;145(4):303-14.

43. Tseng CC, Hu LY, Liu ME, Yang AC, Shen CC, Tsai SJ. Risk of depressive disorders following sudden sensorineural hearing loss: a nationwide population-based retrospective cohort study. J Affect Disord. 2016;197:94-9.

44. Stander VA, Thomsen CJ, Highfill-McRoy RM. Etiology of depression comorbidity in combat-related PTSD: a review of the literature. Clin Psychol Rev. 2014;34(2):87-98.

45. Clifford RE, Baker D, Risbrough VB, Huang M, Yurgil KA. Impact of TBI, PTSD, and hearing loss on tinnitus progression in a US marine cohort. Mil Med. 2019;184(11-12):839-46 https://doi.org/10.1093/milmed/usz016.

46. Hinton DE, Chhean D, Pich V, Hofmann SG, Barlow DH. Tinnitus among Cambodian refugees: relationship to PTSD severity. J Trauma Stress. 2006; 19(4):541-6.

47. Fagelson MA. The association between tinnitus and posttraumatic stress disorder. Am J Audiol. 2007;16(2):107-17.

48. Yovell Y, Sackeim HA, Epstein DG, Prudic J, Devanand DP, McElhiney MC, et al. Hearing loss and asymmetry in major depression. J Neuropsychiatry Clin Neurosci. 1995;7(1):82-9.

49. Aubert-Khalfa S, Granier JP, Reynaud E, El Khoury M, Grosse EM, Samuelian $J$ C, et al. Pure-tone auditory thresholds are decreased in depressed people with post-traumatic stress disorder. J Affect Disord. 2010;127(1-3):169-76.

50. Shalev A, Attias J, Bleich A, Shulman H, Kotler M, Shahar A. Audiological evaluation of nonalcoholic, drug-free posttraumatic stress disorder patients. Biol Psychiatry. 1988;24(5):522-30.

51. Mahapatra SB. Deafness and mental health: psychiatric and psychosomatic illness in the deaf. Acta Psychiatr Scand. 1974;50(6):596-611.

52. Oh JH, Park K, Lee SJ, Shin YR, Choung YH. Bilateral versus unilateral sudden sensorineural hearing loss. Otolaryngol Head Neck Surg. 2007;136(1):87-91.

\section{Publisher's Note}

Springer Nature remains neutral with regard to jurisdictional claims in published maps and institutional affiliations. 